\title{
Influence of Increase of the Occlusal Contact Area on the Tension Generation on Natural Teeth and Adjacent Structures by Finite Element Analysis (FEA)
}

\author{
Da Silva $\mathrm{FM}^{1 *}$, Septímio Lanza $\mathrm{MD}^{2}$, Landre Júnior $\mathrm{J}^{3}$, Seraidarian $\mathrm{PI}^{2}$ and Jansen $\mathrm{WC}^{2}$ \\ ${ }^{1}$ School of Dentistry, Faculty of Health Sciences, University Vale do Rio Doce, Governador Valadares, MG, Brazil \\ ${ }^{2}$ Department of Prosthodontics, School of Dentistry, Catholic University of Minas Gerais, Belo Horizonte, MG, Brazil \\ ${ }^{3}$ Department of Mechanical Engineering, Faculty of Mechanical Engineering, Catholic University of Minas Gerais, Belo Horizonte, MG, Brazil
}

"Corresponding author: Frederico Marques da Silva, DDS, MSc, School of Dentistry, Faculty of Health Sciences, University Vale do Rio Doce, Governador Valadares, MG, Brazil, Tel: +55 31 3823.9541; E-mail: fredmsilva@yahoo.com.br

Rec date: May 16, 2014; Acc date: Jun 17, 2014; Pub date: Jun 19, 2014

Copyright: (c) 2014 Da Silva FM, et al. This is an open-access article distributed under the terms of the Creative Commons Attribution License, which permits unrestricted use, distribution, and reproduction in any medium, provided the original author and source are credited

\begin{abstract}
Background: The increase in the occlusal contact area can generate significant stresses on the occlusal surface of a natural tooth as well as the cervical region and in the alveolar crest around this same tooth edge.

Objective: The objective of this study was to study the influence of the increase of the occlusal contact area on the tension generation on a molar tooth and adjacent structures by three-dimensional finite element models.

Methods: A tridimensional model composed of an inferior molar tooth and surrounding structures was fabricated, and a load of $100 \mathrm{~N}$ was applied over the occlusal surface on pre-set regions according to an ideal occlusal standard that was previously defined for the study. The diameters of the contact spots on the tooth were increased gradually and varied from $0.5 \mathrm{~mm}$ to $1.0 \mathrm{~mm}$ to $1.5 \mathrm{~mm}$. The generated tension over those contact spots was analyzed and quantified on pre-set regions of the occlusal marginal crest: the cervical region and the alveolar crestal bone.
\end{abstract}

Results: The results showed that occlusal contact diameter causes a decrease in the tension level of $33.8 \%$ on the marginal crests, a decrease of $20.7 \%$ on the alveolar rim interface, and an increase of $44.1 \%$ on the cervical region, near the cement enamel junction.

Conclusion: The occlusal contact diameter on the tooth surface had an influence on the tension generated on all analyzed regions.

Keywords: Dental occlusion; Occlusal adjustments; Occlusal contacts; Finite element analysis

\section{Introduction}

Despite the lengthy study and complex assessment of occlusion, its understanding, diagnosis and treatment remain controversial. There are numerous existing philosophies related to this subject, especially regarding the ideal occlusal contact types for individual subjects.

Edward Angle introduced the first description of occlusal tooth relationships in 1899. Later, in 1922, the first significant concept about the ideal functional occlusion was described: bilateral balanced occlusion. With the evolution of occlusal studies and the performance of long-term and complex rehabilitation, a controversy emerged about the suitability of bilateral balanced occlusion in the natural dentition, thereby generating a new concept named unilateral balanced occlusion [1].

It has been mentioned that the stability of human tooth position depends mainly on the resulting interactions of occlusal contacts, not only of pertinent stomatognathic normal function but also of parafunctional habits, such as bruxism [2].Opposing occlusal contacts of the same intensity should provide the teeth and the mandible with mesiodistal and buccolingual stability from the stopper/equalizer contacts and from the A, B, C contacts [3]. In a clinical study, [4] asymmetry in the quantity and distribution of the occlusal contacts between the sides of the dental arch was observed. Thus, those authors concluded that due to the quantity of unstable contacts, intervention involving the occlusal surfaces should be performed to maintain and/or improve the number of those contacts. Similarly, it was mentioned that when the occlusal contacts are distributed irregularly throughout the dental arch, they are capable of promoting dental motion, generating occlusal instability [5]. The variation of an axial force from zero to 30 degrees and inclined on a molar isolated in the arc promotes a significant increase of four times the force supported by the alveolar bone on the side. This can result in tooth mobility and bone loss adjacent to the pressure received [6].

In an ideal occlusion philosophy, to be stable, an occlusal scheme within a balanced cusp-fossa intercuspal position should present at least one occlusal contact per tooth in order to avoid position changes and occlusal interferences $[7,8]$. The contacts among molar teeth occur not only between cusp tips and fossae but also between cusp tips and marginal crests $[1,9,10]$.

Some authors have commented [10,11] that with an increase in intermaxillary closing intensity, the number of occlusal contacts 
Citation: Da Silva FM, Septímio Lanza MD, Landre Júnior J, Seraidarian PI, Jansen WC (2014) Influence of Increase of the Occlusal Contact Area on the Tension Generation on Natural Teeth and Adjacent Structures by Finite Element Analysis (FEA). Dentistry 4: 244. doi: $10.4172 / 2161-1122.1000244$

Page 2 of 5

between teeth increased linearly at the molar region and in a lower proportion the pre-molar and anterior tooth regions. In contrast, the average calculated from the strengths of the flat occlusal contact area remained unchanged from the bite pressure [12].

In physiological occlusion, the posterior teeth come into contact with their antagonists through the punctate areas, a product of the relationship between two convex areas which, by pathologic or physiologic attrition, would be capable of generating abrasion facets, transforming curves into flat surfaces. The occlusal contact area on the dental surfaces is approximately 3 to $4 \mathrm{~mm}^{2}$ in size. With the formation of these facets, the area increases considerably $[9,13]$. Similarly, it was said that the abrasion facets are derived from excessive biomechanical force on the teeth, are considered to be local injuries, [14] and that these forces could also cause distant injuries, such as muscle pain, dental hypersensitivity, and dental mobility [15]. Individuals with a vertical masticatory cycle, similar to the bite of a carnivorous animal, exhibit wear facets that are smaller than those of individuals with a masticatory cycle that is less vertical and more lateral, similar to the bite of an herbivorous animal [13].

Many methods have been used in the study of occlusal dynamics and of the entire stomatognathic system, aiming to achieve a better comprehension of its biomechanics. Many models have been employed, such as the physical model, [1] a wood jawbone and dry human skull, [16] photoelastic resin models, strain gauges, $[17,18]$ and mathematical models of two models of two or three-dimensional finite elements [19].

For some authors, [20] there are three types of studies that could be performed to assess the mandible's behavior. Among those studies, we can isolate the free-body analysis, which offers power vectors and hypothetical direction, providing a closer idea of the nature of the internal forces acting on the structures. The photoelasticity models offer advantages, such as the easy fabrication and visualization of the internal force effects; however, they do not provide a tridimensional view of the distribution of tension. Finite element analysis (FEA) offers the most precision in describing the intensity, direction and duration of the forces and their effects on the degree of deformation and body motion, as well as the distribution of those forces on the internal and external structures of the model. Because of those features, during the last two decades FEA has been established as a very useful tool for assessing the mechanical behavior of biomaterials and human tissues due to its precision and the reliability of its results, demonstrating great precision in describing the intensity and direction of the forces and the degree of deformation and movement of the bodies, [17,20,21] especially considering the difficulties of assessment in vivo. With this method (FEA), some authors [13,17,21-23], have found that variations in vertical and oblique occlusal loads can generate large cervical stresses very near the cementoenamel junction, creating non-carious cervical lesions (abfractions) and bone loss around the necks of the teeth and / or implants.

The literature lacks information about the relationship between the intensity of occlusal contacts and the occlusal contact area, and more studies are necessary to provide a better understanding of the alterations that this relationship could cause on the stomatognathic system-related structures.

Thus, this study sought to assess, by tridimensional finite element analysis, the generation of tension on the natural tooth and its respective neighboring structures, related to an increase in the occlusal contact area. The null hypothesis states that there will be no difference in the generated tension on the natural teeth and on the alveolar rim, independent of the occlusal contact size.

\section{Materials and Methods}

With the objective of researching the influence of the increase in occlusal contact area, a mathematical model composed of an inferior molar and its respective alveolar bone was developed with the specific industrial design software IronCAD version 9.0 (IronCAD, Atlanta, Georgia, USA). This system is parametric design software, aimed at generating complex geometric shapes, enabling work with accuracies of microns, which cannot be achieved by some scanner. The mandibular alveolar bone and the natural tooth, along with their embodied structures, were considered to be a solid body, showing homogeneous and isotropic properties. The material is considered isotropic when the mechanical properties are the same, regardless of the direction in which it is requested and homogeneous when there is a uniformity of material characteristics that shape.

This methodology was idealized in this way, aiming at simplifying the model conception process without compromising the final result. Although the periodontal ligament serves as a fundamental structure in absorbing and transmitting the occlusal forces in vivo, considering its deformation and tension using FEA, this structure should only be generated in a model when it is necessary to illustrate its viscoelastoplastic characteristics [24]. Because the models in this study have linear elastic characteristics that are assessed at definite positions, whose hypothesis is that the deformation of elastic bodies is proportional to the force applied, its construction is dispensable without compromising the proposed test results.

Following the basic created geometry and the assumption of the elastic properties from the diverse structure and its associated materials [25], finite element meshes were independently generated for each anatomic structure using SolidWorks ${ }^{\bullet}$ version Office Premium (Dassault Systèmes SolidWorks Corporation, Concord, Massachusetts, USA) software. For mesh construction, tetrahedral elements were used, with four nodes and three degrees of freedom per node. Therefore, the model was composed of 76,282 elements and 113,429 nodes (Figure 1). The mesh construction takes into account the curvature of the model and this is achieved by the elements used, which are called self-adaptive.

The structures that comprised the model provided free vertical movement and restricted horizontal displacement. This restriction has the objective of avoiding bloc movement after loading.

Static axial charges of $100 \mathrm{~N}$ were applied over the occlusal surface of each generated tooth and were distributed with the same intensity. This load was established on the fact that in the case of linear elastic model deformations obtained is directly dependent on the applied load and follows a linear distribution. Considering this fact and being isotropic and linear elastic and especially a comparative study models the charge is totally irrelevant.

The occlusal contact placement was established in a standardized way, considering that the posterior teeth had complete cusp-fossa and cusp-crest contacts of cuspid-fossa type and cuspid-crest type [3]. In this way, a set of contacts was added on the occlusal surface of the finite element model (FEM); one on each marginal crest (mesial and distal) and three tripoidism-type contacts on the central fossa (Figure 2). 
Citation: Da Silva FM, Septímio Lanza MD, Landre Júnior J, Seraidarian PI, Jansen WC (2014) Influence of Increase of the Occlusal Contact Area on the Tension Generation on Natural Teeth and Adjacent Structures by Finite Element Analysis (FEA). Dentistry 4: 244. doi: $10.4172 / 2161-1122.1000244$

Page 3 of 5

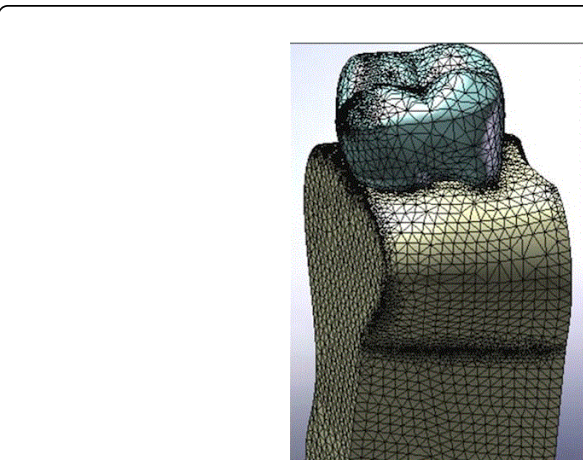

Figure 1: Mesh of the finite element model

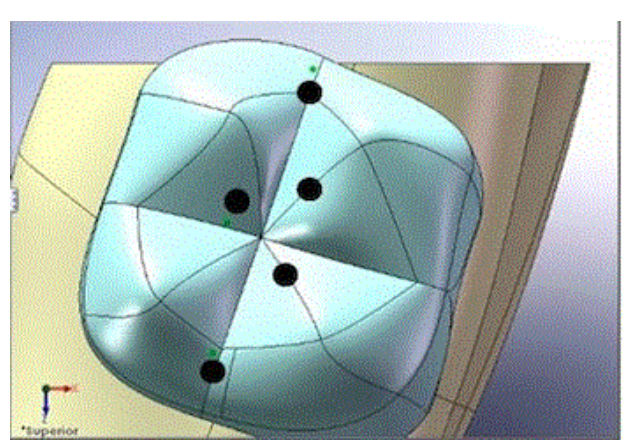

Figure 2: Location of occlusal contact points

For each generated FEM, the diameter of the added contact set varied in each occlusal contact. The occlusal contact diameters that were added over each FEM were $0.5 \mathrm{~mm}, 1.0 \mathrm{~mm}$ and $1.5 \mathrm{~mm}$, which were given the abbreviations FEM-1, FEM-2, FEM-3, respectively.

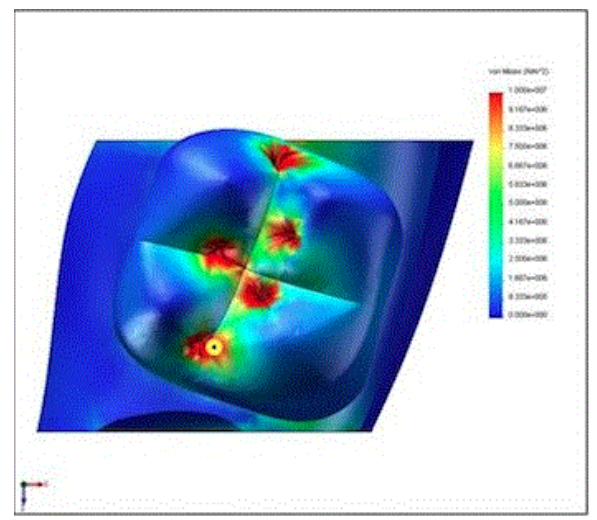

Figure 3: Tension point selected on the occlusal surface

To verify the influence of the diameter of the contact point on the tension generation on the tooth and its associated structures, three different points were randomly selected at specific regions: at the marginal crest region, at the cervical region of the tooth (cementoenamel junction), and at the alveolar crest bone, as marked with a yellow point (Figures 3-5).
The selection of the regions to be analyzed was based on a qualitative interpretation by a color gradient, which represented maximum and minimum value levels of the tension intensity in a region, with red representing the maximum level and blues the minimum level.

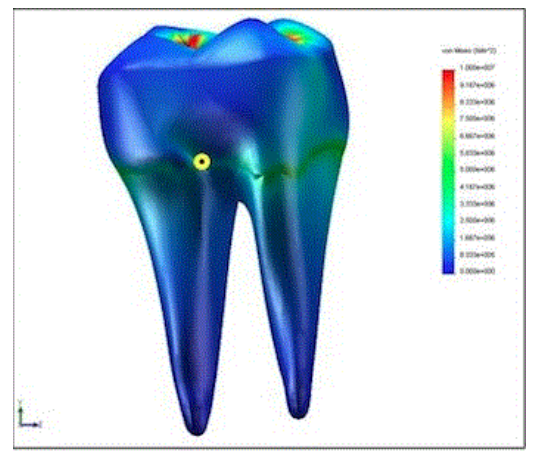

Figure 4: Tension point selected in the cervical region

\section{Results}

The results of the analysis were calculated and obtained using CosmosWorks ${ }^{\oplus}$ software, 2008 version (Dassault Systèmes SolidWorks Corporation, Concord, Massachusetts, USA), which helped to establish graphic and number samples for the study.

Based on these results (Table 1), from the occlusal view of FEM-1, specifically at the selected point at the marginal crest, it was observed that the tension was $2.4 \%$ higher than that of FEM-2, and this value was $30.7 \%$ higher than that of FEM-3. The tension point generated at FEM-1 was $33.8 \%$ higher than that of FEM-3.

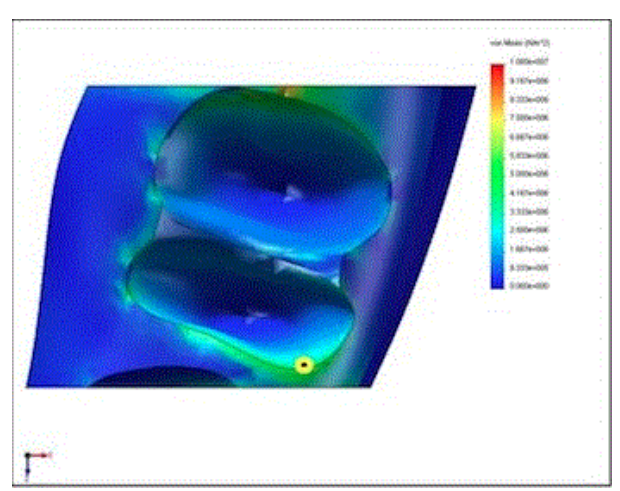

Figure 5: Tension point selected on the alveolar bone crest

\begin{tabular}{|l|l|l|l|}
\hline & Marginal Crest & Cervical Region & Alveolar Ridge \\
\hline FEM-1 & 51 & 3.4 & 6.4 \\
\hline FEM-2 & 49.8 & 4.5 & 5.6 \\
\hline FEM-3 & 38.1 & 4.9 & 5.3 \\
\hline
\end{tabular}

Table 1: shows the generated tensions at the different points of interest selected for this study. The mean pooled values are in $\mathrm{MPa}$ 
Citation: Da Silva FM, Septímio Lanza MD, Landre Júnior J, Seraidarian PI, Jansen WC (2014) Influence of Increase of the Occlusal Contact Area on the Tension Generation on Natural Teeth and Adjacent Structures by Finite Element Analysis (FEA). Dentistry 4: 244. doi: $10.4172 / 2161-1122.1000244$

Page 4 of 5

Following the same pattern, when we analyzed tension at the cervical region, the tension scores were different when compared to those of the previous location. In this case, the model that obtained the highest tension levels was FEM-3, followed by FEM-2 and FEM-1. Comparing the scores, we observed that the tension generated at FEM-3 was $8.8 \%$ higher than that generated at FEM-2 and $44.1 \%$ higher than that generated at FEM-1. In the same way, the tension generated at FEM-2 was $32.3 \%$ higher than at FEM-1.

At the alveolar bony ridge (crest), the scores differed among the three models: the tension at FEM-1 was $14 \%$ higher than at FEM-2 and $20.7 \%$ higher than at FEM-3. FEM-2 presented a tension score that was $5.8 \%$ higher than that of FEM-3.

\section{Discussion}

The objective of this preliminary study was to assess the effects of the occlusal contact diameter on the generation of tension at distinct regions of a "natural" tooth using FEA. We assumed that this different contact diameter might happen clinically in restorative procedures. Following the analysis of results, the null hypothesis was partially rejected due to the proportional differences between contact size and tension increase in certain situations.

The generated structures in this study, such as the alveolar bone and tooth, showed uniform (isotropic) properties [25]. The finite element mesh on the tetrahedral form was adjusted according to the model's requirements, with its nodes exhibiting three degrees of freedom, thereby increasing its precision. The simplification of the FEM technique, specifically the omission of the periodontal ligament, did not diminish the precision of the data generated by these models $[17,21,24,25]$.

FEA studies show that axial charges can generate uniform tension on the tooth roots, showing a higher concentration of tension at the occlusal surface (contact point) and, in a similar way, on the cervical region, close to the cementoenamel junction; the tension in these regions is higher than that generated on the roots [23-25]. These findings agree with those of this study.

The cusp-marginal ridge (mesiodistal stability) and cusp-fossa (buccolingual stability) points of contact were employed in this work in accord with the philosophy of other authors [1-3,19]. It is unanimous among these authors that the principle of ideal occlusion is related to the presence of stable occlusal contacts, simultaneous and bilateral, with the same intensity and direction, and that the forces are directed toward the long axis of the tooth. In contrast, changes in the slope of the occlusal force increase at four times the strain on the alveolar ridge, regardless of the diameter of the occlusal contact [6].

By studying the diameter of the occlusal contact area, it is possible to assess the dental and periodontal conditions and relate them to the parafunctional habits [14]. Thus, the occlusal contact area for the entire mouth should be 3 to $4 \mathrm{~mm}^{2}$ in size. However, with the appearance of wear facets, this area could reach 2 to $4 \mathrm{~mm}^{2}$ per tooth, or it may even extend across the entire surface of the tooth [1]. Despite the fact that the bite force on the tooth is constant and instantaneous, an increase in this variable would cause a linear increase in the size of the occlusal contact area [10-12]. The diameter of the occlusal contact increases due to functional and/or parafunctional masticatory friction, and if there is a need for restorative treatment or occlusal adjustment, it should not introduce a convex shape, nor should it decrease the occlusal contact area [13], according to the results of this study.

\section{Conclusion}

Within the quantitative analysis of the present study and with respect to its limitations, we can conclude that the increase occlusal contacts diameter creates less tension in the region of the alveolar ridge. This increase also increases the probability of causing a noncarious cervical lesion, and the tension levels localized at the marginal crest tend to diminish, minimizing the possibility of tooth fracture. As a suggestions, others researches could add more contact diameters and different shape of teeth.

\section{Acknowledgements}

We would like to thank the Mechanical Engineering Department of the Pontifícia Universidade Católica de Minas Gerais for assistance in the development of this study.

\section{References}

1. Okeson JP (1988) Management of temporomandibular disorders and occlusion. Mosby, St Louis.

2. Ramfjord SP, Ash MM (1983) Occlusion. WB Saunders, Philadelphia.

3. McHorris WH (1985) Occlusal Adjustment via Selective Cutting of Natural Teeth. Int J Periodontics Restorative Dent 5: 8-25.

4. McDevitt WE, Warreth AA (1997) Occlusal contacts in maximum intercuspation in normal dentitions. J Oral Rehabil 24: 725-734.

5. Mahony D (2005) Refining occlusion with muscle balance to enhance long-term orthodontic stability. J Clin Pediatr Dent 29: 93-98.

6. Hood JA, Farak JW, Craig RG (1975) Stress and deflection on three different pontic design. J Prosthet Dent 33: 54-59.

7. Becker CM, Kaiser DA (1993) Evolution of occlusion and occlusal instruments. J Prosthodont 2: 33-43.

8. Wiskott HWA, Belser UC (1995) A rationale for a simplified occlusal design in restorative dentistry: Historical review and clinical guidelines J Prosthet Dent 73: 169-183.

9. Alonso AA, Albertini JS, Bechelli AH (1999) Oclusion and diagnosis in oral rehabilitation. Panamericana, Buenos Aires.

10. Gurdsapsri W, Ai M, Baba K, Fueki K (2000) Influence of clenching level on intercuspal contact area in various regions of dental arch. J Oral Rehabil 27: 239-244.

11. Kumagai H, Suzuki T, Hamada T, Sondang P, Fujitani M, et al. (1999) Occlusal force distribution on the dental arch during various levels of clenching. J Oral Rehabil 26: 932-935.

12. Hidaka O, Iwasaki M, Saito M, Marimoto $T$ (1999) Influence of clenching intensity on bite force balance, occlusal contact area and average bite pressure. J Dent Res 78: 1336-1344.

13. Kim SK, Kim KN, Chang IT, Heo SJ (2001) A study of the effects of chewing patterns on occlusal wear. J Oral Rehabil 28: 1048-1055.

14. Hibi H, Ai M (1997) An optical system for measuring inclination and area of occlusal facets. J Oral Rehabil 24: 673-677.

15. Simon J (2000) Biomechanically-induced dental disease. Gen Dent 48: 598-605.

16. Hatcher DC, Faulkner MG, Hay A (1986) Development of a mechanical and mathematical model to study temporomandibular joint loading. J Prosthet Dent 55: 377-384.

17. Daegling DJ, Hylander WL (2000) Experimental observation, theoretical models and biomechanical interference in the study of mandibular form. Am J Phys Anthropol 112: 541-551.

18. Sakagushi R, Brust EW, Cross M, Delong R, Douglas WH (1991) Independent movement of cusps during occlusal loading. Dent Mater 7: 186-190.

19. Korioth TWP, Romilly DP, Hannam AG (1992) Three-dimensional finite element analysis of the dentate human mandible. Am J Phys Anthropol 88: 69-96. 
Citation: Da Silva FM, Septímio Lanza MD, Landre Júnior J, Seraidarian PI, Jansen WC (2014) Influence of Increase of the Occlusal Contact Area on the Tension Generation on Natural Teeth and Adjacent Structures by Finite Element Analysis (FEA). Dentistry 4: 244. doi: $10.4172 / 2161-1122.1000244$

Page 5 of 5

20. Wakabayashi N, Ona M, Suzuki T, Ygarashi Y (2008) Nonlinear finite element analyses: Advances and challenges in dental applications. J Dent 36: 463-471.

21. Baiamonte T, Abbate MF, Pizzarello F, Lozada J, James R (1996) The experimental verification of the efficacy of MEF to dental implant systems. J Oral Implantol 22: 104-10.

22. Ress JS (2002) The effect of variation in occlusal loading on the development of abfraction lesions: a finite element study. J Oral Rehabil 29: 188-93.
23. Yettran AL, Wright KWJ, Pickard HM (1976) Finite element stress analysis of the crown of normal restored teeth. J Dent Res 55: 1004-11.

24. 24. Ress JS (2001) An investigation into the importance of the periodontal ligament and alveolar bone as supporting structures in finite element studies. J Oral Rehabil 28: 425-32.

25. Menicucci G, Mossolov A, Mozzati M, Lorenzetti M, Preti G (2002) Tooth-implant connection: some biomechanical aspects based on finite element analysis. Clin. Oral Implants Res 12: 334-341. 